\title{
METRIZATION OF SYMMETRIC SPACES AND REGULAR MAPS
}

\author{
HAROLD W. MARTIN
}

\begin{abstract}
A symmetric $d$ for a topological space $R$ is said to be coherent if whenever $\{x(n)\}$ and $\{y(n)\}$ are sequences in $R$ with $d(x(n), y(n)) \rightarrow 0$ and $d(x(n), x) \rightarrow 0$, then $d(y(n), x) \rightarrow 0$. V. Niemytzki and W. A. Wilson have essentially shown that a topological space $R$ is metrizable if and only if $R$ is symmetrizable via a coherent symmetric. Conditions on a symmetric $d$ which are equivalent to $d$ being coherent are established. As a consequence, a theorem of A. Arhangel'skii may be refined by showing that if $f: R \rightarrow Y$ is a quotient map from a metrizable space $R$ onto a $T_{0}$-space $Y$, then $Y$ is metrizable if and only if $f$ is a regular map.
\end{abstract}

For a set $R$, a nonnegative real valued function $d$ on $R \times R$ is called a distance function for $R$ if the following two conditions are satisfied: $d(x, y)=0$ if and only if $x=y$ and $d(x, y)=d(y, x)$. The pair $(R, d)$ is called a distance space where $R$ is a set and $d$ is a distance function for $R$. Two distance functions $d$ and $p$ for a set $R$ are said to be equivalent provided that for any sequence $\{x(n)\}$ in $R$ and any point $x$ in $R$, we have $d(x(n), x) \rightarrow 0$ if and only if $p(x(n), x) \rightarrow 0$. A distance space $(R, d)$ is metrizable provided there exists a metric for $R$ which is equivalent to $d$. In [7], V. Niemytzki calls a distance function $d$ for a set $R$ coherent if whenever $\{x(n)\}$ and $\{y(n)\}$ are sequences in $R$ such that $d(x(n), y(n)) \rightarrow 0$ and $d(x(n), x) \rightarrow 0$, then $d(y(n), x) \rightarrow 0$.

THEOREM 1 (NIEMYTZKI, WILSON [7], [9]). If $d$ is coherent, the distance space $(R, d)$ is metrizable.

A. H. Frink [5] gives a very elegant proof of Theorem 1. Similar metrization theorems and conditions equivalent to coherence may be found in [4], [5], [7], [9].

Let $(R, d)$ be a distance space. A subset $A$ of $R$ is said to be $d$-closed if and only if $d(x, A)>0$ whenever $x \in R-A$. The complements of $d$-closed sets form a topology $T_{d}$ for $R$. Moreover, if $d$ and $p$ are equivalent

Received by the editors October 11, 1971.

AMS 1969 subject classifications. Primary 5435, 5460.

Key words and phrases. Distance function, coherent distance function, distance space, symmetric, coherent symmetric, symmetrizable space, regular map, coherent map.

(. American Mathematical Society 1972 
distance functions for $R$, then $T_{d}=T_{p}$. The triple $\left(R, d, T_{d}\right)$ is called a symmetric space. A topological space $(R, T)$ is said to be symmetrizable provided there exists a distance function $d$ for $K$ such that $T=T_{d}$. When speaking of a distance space as a symmetric space, 've shall call the distance function a symmetric. The concept of a symmetrizable space is due to A. V. Arhangel'skii [3, p. 125]. It is clear from the remarks above that Theorem 1 yields the following: a topological spice $R$ is metrizable if and only if $R$ is symmetrizable via a coherent symmetric. Herein lies the importance of the following theorem.

THEOREM 2. Let $d$ be a symmetric for a topological space $R$. The following four conditions are equivalent:

(1) $d$ is coherent.

(2) If $d(x(n), y(n)) \rightarrow 0$ and $x(n) \rightarrow x$, then $y(n)->x$.

(3) If $d(x(n), A) \rightarrow 0$ where $A$ is compact, then $\{x(n)\}$ has a subsequence which converges to a point of $A$.

(4) If $A$ and $B$ are disjoint subsets of $R$, one of which is closed and the other compact, then $d(A, B)>0$.

Proof. Let $d$ be coherent, $x \in R$ and $S=\{y \in R: d(x, y)<e\}$ where $e$ is some positive real number. Let $A=R-S$ and $B=\{y \in R: d(y, A)=0\}$.

Let $b \in R$ with $d(b, B)=0$. Then there exists a sequence $\{b(n)\}$ in $B$ with $d(b, b(n)) \rightarrow 0$. Choose a sequence $\{a(n)\}$ in $A$ with $d(b(n), a(n)) \rightarrow 0$. Since $d$ is coherent, we have $d(b, a(n)) \rightarrow 0$ so that $d(b, A)=0$. But then $b \in B$ and it follows that $B$ is a closed set. Consequently, we have $x \in$ int $S$.

Let $\{x(n)\}$ be a sequence in $R$ and $x \in R$ such that $d(x(n), x) \rightarrow 0$. Then there exists $e>0$ and a subsequence $\{x(n(i))\}$ of $\{x(n)\}$ such that $x(n(i)) \notin S$ for $i=1,2, \cdots$, where $S=\{y \in R: d(x, y)<e\}$. But, as seen above, $x \in$ int $S$, so that $x(n(i))+\rightarrow x$, whence $x(n) \rightarrow x$. By contraposition, we see that if $x(n) \rightarrow x$, then $d(x(n), x) \rightarrow 0$. That (2) holds is now evident.

Now assume that $d$ satisfies condition (2). Let $\{y(n)\}$ be an arbitrary convergent sequence in $R$ with $y(n) \rightarrow y$. Let $Y=\{y\} \cup\{y(1), y(2), \cdots\}$ and let $z \in R-Y$. Suppose that $d(z, Y)=0$. Then there exists a subsequence $\{y(n(i))\}$ of $\{y(n)\}$ with $d(y(n(i)), z) \rightarrow 0$. For $i=1,2, \cdots$, let $z(i)=z$. Then we have $d(y(n(i)), z(i)) \rightarrow 0$ and $y(n(i)) \rightarrow y$. Since $d$ satisfies (2), this implies that $z(i) \rightarrow y$, which contradicts the fact that $R$ is a $T_{1}$-space and $y \neq z$. Therefore, $d(z, Y)>0$, that is, $Y$ is a closed set.

Let $B$ be any nonclosed subset of $R$. There exists $x \in R-B$ with $d(x, B)=0$. Choose a sequence $\{x(n)\}$ in $B$ with $d(x, x(n)) \rightarrow 0$ and $x(n) \neq$ $x(m)$ for $n \neq m$. By the previous paragraph, the set $\{x\} \cup\{x(1), x(2), \cdots\}$ is closed in $R$ so that $\{x(1), x(2), \cdots\}$ is closed in the subspace $B$. Similarly, $\{x(n), x(n+1), \cdots\}$ is closed in $B$ for $n=2,3, \cdots$. Let $H_{n}=B-$ $\{x(n), x(n+1), \cdots\}$ for $n=1,2, \cdots$. Each set $H_{n}$ is open in the relative 
topology for $B$ and $\left\{H_{n}: n=1,2, \cdots\right\}$ covers $B$. Clearly $\left\{H_{n}\right\}$ has no finite subcover so that $B$ is not compact. It follows that compact subsets of $R$ are closed.

We are now in a position to show that $d$ satisfies (3). Let $A$ be a compact subset of $R$ and let $\{x(n)\}$ be a sequence in $R$ with $d(x(n), A) \rightarrow 0$. Since $A$ is a closed subset of $R, A$ is itself a symmetrizable space, and therefore sequentially compact. Choose a sequence $\{a(n)\}$ in $A$ with $d(x(n), a(n)) \rightarrow 0$. There exists a subsequence $\{a(n(i))\}$ of $\{a(n)\}$ and a point $a \in A$ with $a(n(i)) \rightarrow a$. Since $d$ satisfies (2), it follows that $x(n(i)) \rightarrow a$, that is, $d$ also satisfies (3).

If $d$ does not satisfy (4), then there exists a compact set $A$ and a closed set $B$, disjoint from $A$, with $d(A, B)=0$. Choose a sequence $\{x(n)\}$ in $B$ with $d(x(n), A) \rightarrow 0$. Any convergent subsequence of $\{x(n)\}$ must converge to a point of $B$ so that $d$ does not satisfy (3). It follows that if $d$ satisfies (3), then $d$ also satisfies (4).

Finally, let $d$ satisfy condition (4). If $A$ is a compact set and $x \in R-A$, then $d(x, A)>0$ since the singleton set $\{x\}$ is closed and disjoint from $A$. Consequently, compact subsets of $R$ are closed. Let $\{x(n)\}$ and $\{y(n)\}$ be sequences in $R$ and $x \in R$ such that $d(x(n), y(n)) \rightarrow 0$ and $d(x, x(n)) \rightarrow 0$. Suppose that $d(x, y(n)) \rightarrow 0$. Without loss of generality we may suppose that there exists $e>0$ such that $d(x, y(n))>e$ for $n=1,2, \cdots$. Let $Y=$ $\{y(1), y(2), \cdots\}$ and $X=\{x\} \cup\{x(n): x(n) \notin Y\}$. Since $X$ is compact, disjoint from $Y, d(X, Y)=0$ and $d$ satisfies (4), $Y$ cannot be closed. Let $y \in R-Y$ with $d(y, Y)=0$. Choose a subsequence $\{y(n(i))\}$ of $\{y(n)\}$ such that $d(y, y(n(i))) \rightarrow 0$ as $i \rightarrow \infty$. Let $Y^{\prime}=\{y\} \cup\{y(n(i)): i=1,2, \cdots\}$. Since $Y^{\prime}$ is compact, it is also closed. Let $X^{\prime}=X-\{y\}$. Then $X^{\prime}$ is compact and disjoint from $Y^{\prime}$ so that $d\left(X^{\prime}, Y^{\prime}\right)>0$. But $d(x(n), y(n)) \rightarrow 0$ implies that $d\left(X^{\prime}, Y^{\prime}\right)=0$. The supposition that $d(x, y(n))+\rightarrow 0$ has led to a contradiction; consequently, $d(x, y(n)) \rightarrow 0$ and $d$ is coherent, completing the proof.

A symmetric $d$ for a topological space $R$ is said to satisfy condition A provided that $d(F, K)>0$ whenever $F$ and $K$ are disjoint closed subsets of $R$, at least one of which is compact. A Hausdorff space is metrizable if and only if it is symmetrizable via a symmetric which satisfies condition A [3, Theorem 3.1]. This result also follows immediately from Theorems 1 and 2 above. The following example, due to Peter Harley, shows that there exist symmetrics which satisfy condition A but which are not coherent. Let $N$ be the set of all positive integers. For $n, m \in N$ with $n \neq m$, define $d(n, m)=(|n-m|)^{-1}$. The symmetric $d$ generates the cofinite topology for $N$, that is, the $d$-closed sets of $N$ are the finite subsets of $N$ and $N$ itself. The symmetric $d$ clearly satisfies condition A but $d$ is not coherent since $(N, d)$ is not metrizable. 
We say that a map $f: R \rightarrow Y$ from a metrizable space $R$ onto a space $Y$ is coherent if $R$ is symmetrizable by a symmetric $d$ such that if $\{x(n)\}$ and $\{y(n)\}$ are sequences in $R$ with $d(x(n), y(n)) \rightarrow 0$ and $f(x(n)) \rightarrow y$, then $f(y(n)) \rightarrow y$. Every coherent map is continuous. A map $f: R \rightarrow Y$ from a metrizable space $R$ onto a topological space $Y$ is said to be regular provided there exists a compatible metric $p$ for $R$ such that if $y \in V$ where $V$ is open in $Y$, then there exists an open neighborhood $W$ of $y$ for which $p\left(f^{-1}[W], R-f^{-1}[V]\right)>0$. Any continuous map from a metrizable space onto a metrizable space is regular [3, p. 134]. Every regular map is coherent, as seen in the proof of the following:

Lemma. Let $f: R \rightarrow Y$ be a function from a metrizable space $R$ onto a metrizable space $Y$. Then, the following are equivalent:

(1) $f$ is continuous.

(2) $f$ is regular.

(3) $f$ is coherent.

Proof. Let $p$ be a metric for $R$ and $d$ be a metric for $Y$. For $a, b \in R$ define $s(a, b)=p(a, b)+d(f(a), f(b))$. Since $f$ is continuous, the metrics $p$ and $s$ are equivalent. $f$ is regular via $s$. Now assume that $f$ is a regular map by virtue of a metric $p$ for $R$. Let $\{x(n)\}$ and $\{y(n)\}$ be sequences in $R$ with $p(x(n), y(n)) \rightarrow 0$ and $f(x(n)) \rightarrow y$ for some $y \in Y$. Let $V$ be an open set in $Y$ which contains $y$. Since $f$ is regular by virtue of $p$, there exists an open neighborhood $H$ of $y$ such that $p\left(f^{-1}[H], R-f^{-1}[V]\right)=e>0$. Since $f(x(n)) \rightarrow y$, the sequence $\{x(n)\}$ is eventually in the set $f^{-1}[H]$. Since $p(x(n), y(n)) \rightarrow 0$, it follows that the sequence $\{y(n)\}$ must eventually be in the set $f^{-1}[V]$, that is $\{f(y(n))\}$ is eventually in $V$ so that $f(y(n)) \rightarrow y$, proving that $f$ is a coherent map. That (3) implies (1) is almost immediate, completing the proof.

Let $f: R \rightarrow Y$ be a quotient map from a metrizable space $R$ onto a $T_{1}$-space $Y$. In [3, p. 134], Arhangel'skii established the following results: $Y$ is metrizable if and only if $f$ is regular and pseudo-open; and, if $Y$ is Hausdorff, then $Y$ is metrizable if and only if $f$ is regular. The following sharpens these results and completes the solution to a problem raised in $[1$, p. 368].

THEOREM 3. Let $f: R \rightarrow Y$ be a quotient map from a metrizable space $R$ onto a $T_{0}$-space $Y$. Then, the following are equivalent:

(1) $Y$ is metrizable.

(2) $f$ is a regular map.

(3) $f$ is a coherent map.

Proof. That (1) implies (2) and (2) implies (3) follows from the Lemma. Therefore, assume that $f$ is a coherent map by virtue of a symmetric $p$ for $R$. Define a function $d$ on $Y \times Y$ by $d(x, y)=p\left(f^{\cdots 1}(x), f^{-1}(y)\right)$. 
Since $Y$ is $T_{0}$ and $f$ is a coherent map via $p$, we have $d(x, y)>0$ for $x \neq y$, that is, $d$ is a distance function. Let $Q$ denote the quotient topology for $Y$ relative to $f$ and $T_{d}$ denote the topology consisting of the complements of $d$-closed sets. Since $f$ is continuous with respect to $T_{d}$, we have $T_{d} \subset Q$. Let $A$ be closed in the space $(Y, Q)$ and let $x \in Y-A$. If $d(x, A)=0$, then there exist sequences $\{x(n)\}$ in $f^{-1}(x)$ and $\{a(n)\}$ in $f^{-1}[A]$ with $p(a(n), x(n)) \rightarrow 0$. But then $f(a(n)) \rightarrow x$ in $(Y, Q)$ since $f$ is coherent, contradicting the assumption that $A$ is closed in $(Y, Q)$. We must have $d(x, A)>0$, that is, $A$ is closed in $\left(Y, T_{d}\right)$. It follows that $Q=T_{d}$, that is, $Y$ is symmetrizable via the symmetric $d$.

Let $\{x(n)\}$ and $\{y(n)\}$ be sequences in $Y$ and $x \in Y$ such that $d(x(n), y(n)) \rightarrow 0$ and $x(n) \rightarrow x$. There exist sequences $\left\{x^{\prime}(n)\right\}$ and $\left\{y^{\prime}(n)\right\}$ in $R$ with $p\left(x^{\prime}(n), y^{\prime}(n)\right) \rightarrow 0$ where $f\left(x^{\prime}(n)\right)=x(n)$ and $f\left(y^{\prime}(n)\right)=y(n)$. Then $f\left(x^{\prime}(n)\right) \rightarrow x$ and since $f$ is a coherent map via $p, f\left(y^{\prime}(n)\right) \rightarrow x$, that is, $y(n) \rightarrow x$. It follows from Theorem 2 that $d$ is a coherent symmetric. The metrizability of $Y$ now follows by Theorem 1, completing the proof.

A map $f: R \rightarrow Y$ is said to be proper if $f^{-1}[A]$ is compact whenever $A$ is compact in $Y$. The following is easy to verify: Let $Y$ be a topological space in which compact subsets are closed; if $f: R \rightarrow Y$ is a proper map from a metrizable space $R$ onto $Y$, then $f$ is a coherent map. As an immediate consequence we have that the proper quotient image of a metric space is metrizable. In fact, if $f: R \rightarrow Y$ is a proper quotient map from a metrizable space $R$ onto a space $Y$, then since $Y$ is metrizable, by a theorem of G. T. Whyburn [10] or by Theorem 2.15 of [2], $f$ is actually a closed map. In short, proper quotient maps on metric spaces are always perfect maps. Therefore, Theorem 3 yields an easy proof of the well-known Morita-Hanai-Stone Theorem [6], [8] that the perfect image of a metric space is metrizable.

I would like to express my indebtedness to my friend Peter Harley for several helpful conversations during the preparation of this paper.

\section{REFERENCIS}

1. A. V. Arhangel'skiī, Factor mappings of metric spaces, Dokl. Akad. Nauk SSSR 155 (1964), 247-250 = Soviet Math. Dokl. 5 (1964), 368-371. MR 29 \#587.

2. - Bicompact sets and the topolog! of spaces, Trudy Moskov. Mat. Obšč. 13 (1965), 3--55 = Trans. Moscow Math. Soc. 13 (1965), 1 -62. MR 33 \#3251.

3. - - Mappings and spaces, Uspehi Mat. Nauk 21 (1966), no. 4(130), 133-184= Russian Math. Surveys 21 (1966), no. 4, 115-162. MR 37 \#3534.

4. E. W. Chittenden, On the metrization problem and related problems in the theory of abstract sets, Bull. Amer. Math. Soc. 33 (1927), 13--34.

5. A. H. Frink, Distance functions and the metrization prohlem, Bull. Amer. Math. Soc. 43 (1937), 133 142. 
6. K. Morita and S. Hanai, Closed mappings and metric spaces, Proc. Japan Acad. 32 (1956), 10-14. MR 19, 299.

7. V. Niemytzki, On the third axiom of metric spaces, Trans. Amer. Math. Soc. 29 (1927), 507-513.

8. A. H. Stone, Metrizability of decomposition spaces, Proc. Amer. Math. Soc. 7 (1956), 690-700. MR 19, 299.

9. W. A. Wilson, On semi-metric spaces, Amcr. J. Math. 53 (1931), 361-373.

10. G. T. Whyburn, Directed families of sets and closedness of functions, Proc. Nat. Acad. Sci. U.S.A. 54 (1965), 688-692. MR 32 \#435.

Department of Mathematics, University of South Carolina, Columbia, South CAROLINA 29208 